\title{
RECIENTE EVOLUCIÓN DE LOS HOGARES UNIPERSONALES EN ESPAÑA
}

\author{
UNA APROXIMACIÓN SOCIOLÓGICA \\ THE RISE OF SINGLE-PERSON HOUSEHOLDS IN SPAIN. \\ A SOCIOLOGICAL APPROACH
}

\author{
Francisco Alberto Vallejo Peña \\ favallejo@uma.es
}

Artículo Recibido: 20/03/2016

Artículo Aceptado: 30/05/2016

\begin{abstract}
Resumen
La proliferación de hogares unipersonales se ha convertido en un rasgo destacable de las sociedades occidentales contemporáneas. España contaba -hasta los 80- con hogares habitados por extensos núcleos familiares, pero el empuje de su proceso modernización y las nuevas tendencias sociodemográficas la han llevado, desde entonces, a un continuo incremento de sus hogares solitarios. El presente trabajo resume la evolución reciente del fenómeno en España en base a datos del INE y de la OCDE, entre otros. Posteriormente se abordan sus implicaciones y repercusiones sociológicas, tratando de conectar el fenómeno con la evolución de la institución familiar, el mercado de trabajo, así como con las nuevas relaciones socioeconómicas que impone la modernidad. Los resultados nos invitan a pensar que los sujetos se ven arrastrados a la vida solitaria, más por un conjunto de factores contextuales, que por el deseo de vivir en soledad bajo la influencia de los nuevos procesos de individuación.
\end{abstract}

Palabras clave: soledad, tercera edad, solteros, vivienda, familia, modernidad.

\begin{abstract}
The rise of single-person households has become a notable feature of contemporary Western societies. Spain had up to the 80 - to homes inhabited by large families, but the thrust of its modernization process and new demographic trends have led a steady increase in their solitary homes. This paper summarizes recent developments in the phenomenon in Spain based on data from the INE and OECD, among others. Later
\end{abstract}

WPS RI-SHUR, nํㅜ, 2016, vol.1, ISSN: 2387-1768 


\title{
WPSReview International on Sustainable
}

\author{
Housing and Urban Renewal (RI-SHUR)
}

sociological implications could connect the phenomenon with the evolution of the family institution, the labor market and the new socioeconomic relations imposed by modernity. The results invite us to think that the subjects are drawn to the solitary life of a set of contextual factors, and not by the desire to live alone under the influence of the new processes of individuation.

Keywords: Ioneliness, elderly, singles, housing, family, modernity.

\section{Planteamiento de la investigación}

El aumento de los hogares unipersonales se ha convertido en un fenómeno extendido dentro del mundo occidental desarrollado. España no es una excepción en este sentido y mostró un impetuoso ascenso en esta forma de vida en los 90 y que continúa a fuerte ritmo hasta la actualidad. Esta tendencia global de los hogares solitarios responde tanto a los propios rasgos socioeconómicos de la modernidad, como a la consecuente evolución de sus valores. Entre los fenómenos que subyacen destaca notoriamente la individuación, con obvio reflejo en las formas de vida en solitario (Bauman, 2005; Sennet, 2000). Asimismo, la crisis de la familia como institución, las nuevas alternativas al modelo tradicional y el creciente fracaso de las parejas -de hecho o de derecho- han influido en el aislamiento que reflejan estos hogares. Por lo tanto, los científicos sociales manifiestan su preocupación por las repercusiones psicosociales del nuevo escenario. Así lo manifiesta el propio Sennet, que entiende que ante nuestra tendencia natural a relacionarnos con el resto de seres humanos, el creciente materialismo nos separa gradualmente de ellos. Para los científicos sociales la búsqueda de sentido del fenómeno es obligada (Barroso et al. 2016), ya que sea cual sea la configuración del orden social que se dé, las personas se necesitan las unas a las otras. Frente a ello, en la modernidad estamos anteponiendo el peso de la decisión personal: la singularidad por encima de todo (Becker, 1987). Asimismo, Ulrich Beck (2002) señala que en la sociedad actual prevalecen las opciones y decisiones individuales frente a las "verdades" de la socialización. Escoger nuestro propio camino -por encima de familiares y amigos- es importante para nosotros, además de todo un rasgo de la interpretación occidental de la modernidad.

Esta tendencia se inició en los 60 -fundamentalmente en países nórdicos y anglosajones-, y desde entonces ha tenido una gradual difusión al resto de los países desarrollados (Roussel, 1986). En el caso español el fenómeno es algo más tardío, su ascenso (estadístico) se inició en la década de los 80 y se ha mantenido a fuerte ritmo hasta la actualidad. Contamos con 4,2 millones de hogares unipersonales sobre un total de 16,8 millones de hogares (INE, 2014). Estos albergan al 10\% de la población (4,5 millones de personas). Entre los "solitarios" predominan las mujeres (2,4 millones), y dentro de ellas la tasa sube intensamente a partir de los 65 años. De esta forma,

WPS RI-SHUR, nำ, 2016, vol.1, ISSN: 2387-1768 


\title{
WPSReview International on Sustainable
}

\author{
Housing and Urban Renewal (RI-SHUR)
}

podemos afirmar que las ancianas se han convertido en toda una tipología del hogar unipersonal español, ya que acaparan el $32 \%$ sobre el total (INE, 2014).

Para la interpretación del fenómeno resultan esenciales las aportaciones de Bauman (2005) sobre el proceso de construcción de una sociedad líquida. A la postre, esto es un factor clave para entender la modernidad. El proceso de industrialización en la sociedad occidental nos ha llevado, gradualmente, a desplazar las relaciones de carácter primario que resultaban antaño esenciales tanto para nuestra supervivencia diaria como para la construcción de nuestra identidad. El utilitarismo creciente va deteriorando tales vínculos, reduciendo y limitando nuestras relaciones primarias. Aspectos como el amor y la amistad no quedan, desafortunadamente, inmunes al creciente fenómeno. Por otra parte, el incremento en la intensidad del intercambio de bienes y servicios ha proyectado las relaciones secundarias como sustitutivas de estas. Para Bauman, además, el boom de la economía digital ha disparado estas posibilidades. Por tomar un claro exponente, debemos considerar la amplia gama de intercambios relacionales que actualmente reemplazan facetas como la relación amorosa tradicional. Ahora, el amor líquido nos permite vivir estas experiencias obviando el fuerte compromiso emocional que implicaban.

La soledad, entonces, se plantea como un fenómeno contemporáneo va más allá de una situación que ha sido achacada a los ancianos. La nueva generación Y identificada como los nacidos después de 1985- valora la soledad o, al menos, una de sus facetas, la que le permite disfrutar de la elección individual, así como eludir los siempre difíciles compromisos con sus semejantes. ¿Es el hogar unipersonal una clara plasmación de este fenómeno? Sin duda, aunque como veremos en el desarrollo de este trabajo, otros tantos factores median en el asentamiento del hogar solitario como modelo occidental (y español).

\section{Objetivos y metodología}

El presente trabajo pretende retratar la evolución de los hogares solitarios en la España de los últimos 40 años (se han triplicado en el periodo), reflexionando sobre sus causas e identificando sus repercusiones sociales. En mi enfoque le doy particular importancia a la evidente conexión del fenómeno con los cambios en la institución familiar (tamaño de la unidad, fragmentación, nuevas formas de unión, divorcios, situación de los ancianos y soledad).

Para tal fin se expone un análisis descriptivo de la evolución de los hogares unipersonales en España en los últimos tiempos. Este incluye una presentación de carácter longitudinal de las variables: tasa de hogares unipersonales (España y OCDE), distribución de los hogares según el número de miembros, estructura de los hogares unipersonales (pirámide de la población de estos hogares), distribución de los hogares unipersonales según el estado civil, distribución según el nivel educativo de

WPS RI-SHUR, n3, 2016, vol.1, ISSN: 2387-1768 


\section{WPSReview International on Sustainable \\ Housing and Urban Renewal (RI-SHUR)}

sus ocupantes, régimen en propiedad/alquiler y proyección del tamaño medio del hogar (hasta 2029).

Posteriormente se contrastan los resultados de tal análisis, con algunas de las aportaciones teóricas recientes más relevantes sobre el fenómeno (Barroso et al. 2016; Lim, 2015, entre otros). De esta forma buscamos una lectura de las cifras de forma paralela a las recientes construcciones teóricas en el campo.

Para la realización del estudio se han tomado como referente datos de 2014 , si bien en algunos casos -por barreras de acceso- se usan datos de los años anteriores como referencia tratando de representar la actualidad. Por otra parte, con pretensiones longitudinales se han recogido datos desde el año 1991. A continuación se exponen las principales fuentes de las que se han obtenido los datos para el estudio:

- Instituto Nacional de Estadística (Censo de Población y Viviendas).

- OCDE (banco de datos sobre familia).

- Encuesta de Opiniones y Actitudes hacia la Familia (OPAFAM).

\section{Los hogares unipersonales en España: reciente evolución en cifras}

La globalización del fenómeno de los hogares unipersonales está ya enormemente extendida dentro del mundo desarrollado. En realidad podemos afirmar que España está en la línea marcada por los países miembros de la OCDE. Actualmente se encuentra cercano al promedio del conjunto $(26.9 \%$ por $25 \%$ del caso español en 2014). Es importante matizar, en este caso, que España está entre los países de modernización tardía y su arranque (años 80 ) es notoriamente posterior a los países pioneros (60 y 70). Se expone el listado completo en la Tabla 1.

Tabla 1. Porcentaje de hogares unipersonales en la OCDE

\begin{tabular}{|l|l|l|l|l|l|l|l|}
\hline Australia & 26,5 & Finlandia & 37,3 & Corea & 24 & Portugal & 17,3 \\
\hline Austria & 33,5 & Francia & 31,0 & Letonia & 25,0 & Rumania & 18,9 \\
\hline Bélgica & 31,6 & Alemania & 35,8 & Lituania & 28,7 & $\begin{array}{l}\text { EsSlovaquia } \\
\text { republic }\end{array}$ & 19,4 \\
\hline Bulgaria & 22,7 & Grecia & 19,7 & Luxemburgo & 29,3 & Eslovenia & 21,9 \\
\hline Canadá & 26,8 & Hungría & 26,2 & México & 7,6 & España & $\mathbf{2 5}$ \\
\hline R. Checa & 30,3 & Islandia & 30,7 & Holanda & 33,6 & Suiza & 36,0 \\
\hline Chipre & 16,0 & Irlanda & 21,6 & N. Zelanda & 22,6 & U. K. & 30,2 \\
\hline
\end{tabular}

WPS RI-SHUR, n3, 2016, vol.1, ISSN: 2387-1768 


\section{WPSReview International on Sustainable}

\section{Housing and Urban Renewal (RI-SHUR)}

\begin{tabular}{|l|l|l|l|l|l|l|l|}
\hline Dinamarca & 36,8 & Italia & 24,9 & Noruega & 37,7 & $\begin{array}{l}\text { Estados } \\
\text { Unidos }\end{array}$ & 27,3 \\
\hline Estonia & 33,5 & Japón & 29,5 & Polonia & 24,8 & OCDE & 26.9 \\
\hline
\end{tabular}

Fuente: OCDE (2014)

El hogar español muestra una fisonomía en la que predomina la situación de dos miembros. Tal configuración ya se impuso en los años 90. De hecho, considera siendo la tipología prioritaria (34,2\% en 2014 , según el INE), a pesar del vertiginoso aumento de los hogares unipersonales en los últimos tiempos: se han duplicado entre $1991 \mathrm{y}$ 2014 (13,3\% frente a 25). Si agrupamos las categorías de una y dos personas recogeremos a la mayoría de nuestros hogares. Por lo tanto, el 59,2\% de nuestros hogares cuentan con solo uno o dos miembros, siendo esto un reflejo muy palpable de la "atomización" de la sociedad española y de la pérdida de peso de la familia nuclear como eje del hogar (ver tabla 2).

Tabla 2. Distribución de los hogares según el número de personas en España (porcentajes)

\begin{tabular}{l|l|l|l|l|l|l}
\hline & & 1 pers. & 2 pers. & 3 pers. & 4 pers. & $\begin{array}{l}5 \text { pers. y } \\
\text { más }\end{array}$ \\
\hline & 2014 & 25 & $\mathbf{3 4 , 2}$ & 20,5 & 16,7 & 3,6 \\
\hline \multirow{3}{*}{ España } & 2011 & 23,2 & $\mathbf{3 0 , 1}$ & 21,7 & 18,5 & 6,5 \\
\cline { 2 - 7 } & 2001 & 20,3 & $\mathbf{2 5 , 2}$ & 21,2 & 21,5 & 11,8 \\
\cline { 2 - 7 } & 1991 & 13,3 & $\mathbf{2 3 , 2}$ & 20,6 & 23,0 & 19,8 \\
\hline
\end{tabular}

Fuente: Censos de Población y Viviendas (INE).

En consecuencia el promedio de miembros por hogar se está reduciendo gradualmente a medida que los hogares unipersonales y los hogares de 2 personas se consolidan como modelo social. En España también se reduce a 2,4 personas en 2014 (gráfico 2), mostrando una sustancial caída desde la cifra de 3,6 personas en 1981. Tal decrecimiento no es una sorpresa y responde a los patrones observados en el resto de los países de la OCDE. 
Gráfico 2. Evolución del número medio de miembros por hogar en España

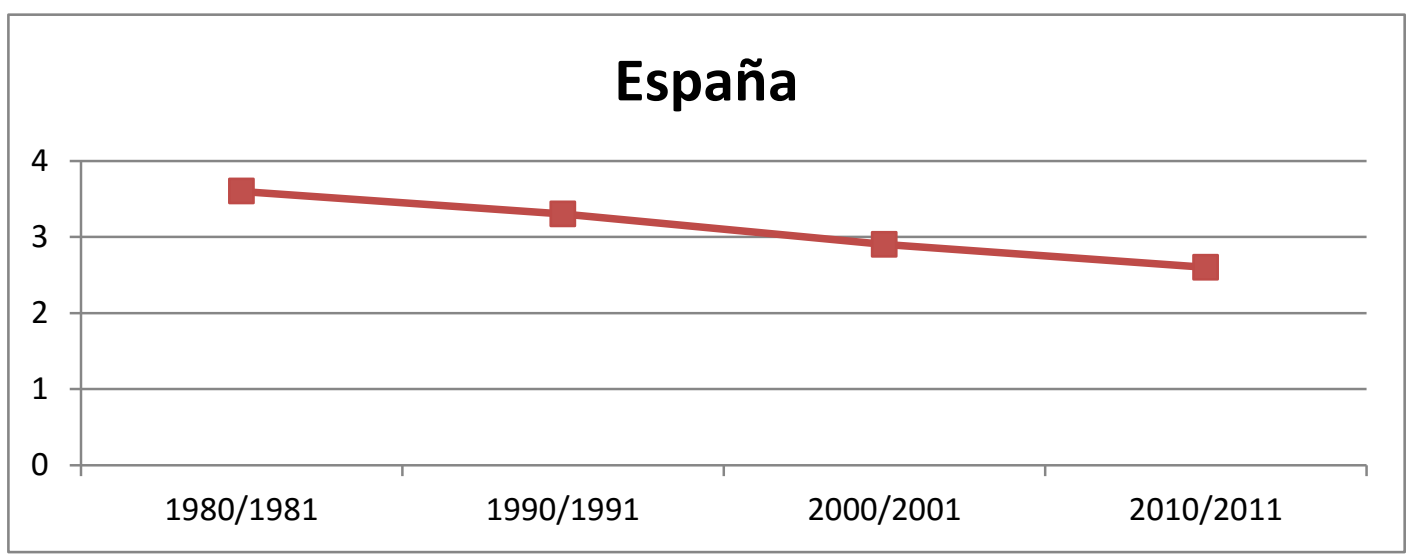

Fuente: Censos de Población y Viviendas (INE).

\section{4. ¿Quiénes habitan nuestros hogares unipersonales?}

Para dar respuesta a esta cuestión resulta ineludible abordar las variables de edad y género. En este ámbito debemos centrarnos en las edades superiores a los 50 años, desde tal punto el incremento de residentes solitarios es notable. Dentro de este grupo destacan notablemente las mujeres, de ahí la tipología señalada en la introducción del artículo, que destacaba una importante concentración en el grupo de tercera edad femenino (mayores de 65 años). La visualización de la pirámide de población de los "solitarios" nos muestra muy claramente la tendencia. El aumento se da desde los 50 años, pero además se puede observar una intensa progresión a partir de los 70, y mucho más notable en el género femenino. El mensaje sociológico trasmitido por el conjunto de los datos es muy relevante desde la perspectiva de Barroso et al. (2016), ya que interpretan situaciones predominantes de "nido vacio". En la mayoría de los casos no se rehusó a la convivencia, más bien se imponen los proyectos familiares y de pareja fracasados, que además -en tales edades- no presentan cambios en perspectiva: el vivir solo después de los 50 se presenta como una circunstancia cercana a lo definitivo por los escasos proyectos de convivencia que se emprenden a partir de esta edad (este no es el caso de los "solitarios" entre los 20 y los 40, que requieren otra lectura por contar con perspectivas de cambiar su situación). En el caso español los hogares unipersonales ocupados por mayores de 65 años experimentaron un aumento del 28,6 \% en la década 2001-2011 (datos del INE, ver en tabla 5). Este es un dato particularmente preocupante, por las ineludibles necesidades $y$ WPS RI-SHUR, nํㅜ, 2016, vol.1, ISSN: 2387-1768 


\section{WPSReview International on Sustainable \\ Housing and Urban Renewal (RI-SHUR)}

responsabilidades implicadas por tal grupo de edad. Las reacciones institucionales no deben hacerse esperar ante un fenómeno que continuará en aumento, tanto por la extensión de las conductas individualistas como por el continuo aumento de la esperanza de vida en España.

Al descender en edades la pirámide española, observamos como el sesgo se produce ahora hacia la izquierda, hacia el lado de los hombres. La tardía edad a la que ellos contraen matrimonio (33) permite observar un incremento notable de "solitarios" al acercarse a la treintena, que esperan modificar su situación en muchos casos. La descripción del grupo que va de los 35 a los 49 estaría compuesto por una amalgama de separados, divorciados, solteros de larga duración y viudos, a los que se han ido sumando en los últimos años los LAT (Living Apart Together). En España han proliferado notoriamente en la última década las relaciones de pareja sin convivencia física, que se sitúan en torno al 8\% (amplio análisis en Ayuso, 2012).

En resumen, en España I distribución de los "solitarios" está notoriamente segmentada por las variables de género y edad, dadas las diferencias en los patrones sociológicos que ambas implican y su directa plasmación en las formas de vida (diferencias generacionales, cambios en el mercado de trabajo, nuevas tendencias en el ámbito de la pareja). El rango 75-79 años presentó el mayor número de 317 mil hogares (ver gráfico 3 y tabla 4), con particular peso en el caso de las mujeres. Este subgrupo comparte el peso con los hombres entre 30 y 49 años, que ensancha la pirámide por la zona inferior hacia la izquierda. 


\section{WPSReview International on Sustainable \\ Housing and Urban Renewal (RI-SHUR)}

Gráfico 3. Estructura de los Hogares unipersonales de España (2014)

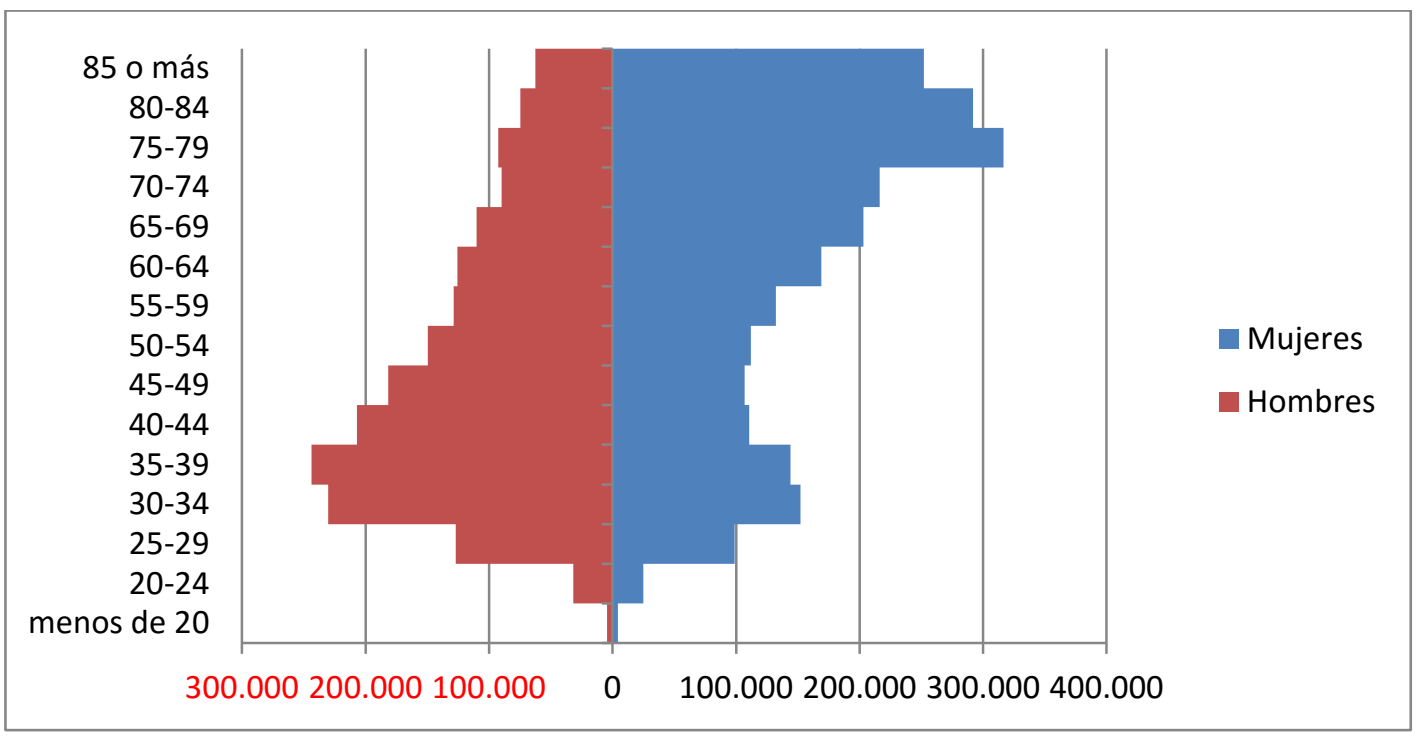

Fuente: Jeongju con datos del Censos de Población y Viviendas (INE)

Tabla 4. Hogares de una persona con 65 años o más (unidad: mil hogares, \%)

\begin{tabular}{l|l|l|l|l}
\hline & $2000 / 2001$ & $2010 / 2011$ & $\begin{array}{l}\text { Variación } \\
\text { absoluta }\end{array}$ & $\begin{array}{l}\text { Variación } \\
\text { Relativa }\end{array}$ \\
\hline España & 1.359 & 1.709 & 351 & $25.8 \%$ \\
\hline
\end{tabular}

Fuente: Censos de Población y Viviendas 2011 Datos detallados (INE)

Abordando el estado civil de los ocupantes de los hogares unipersonales, el estatus que ha presentado mayor incremento es divorciado $(283,5 \%)$, seguido de casado $(53,5 \%)$, soltero $(32,5 \%)$ y viudo $(20,1 \%)$, según datos del INE 2011 (ver tabla 5$)$. En términos de aumento del número de los hogares unipersonales, los solteros aumentaron en 648000 mientras que los divorciados fueron 304000 en tan solo 10 años (2001-2011). El incremento de los hogares unipersonales para el estado civil de Divorciado implica, por lo tanto, una variación muy notoria del 3,7\% al 9,8\% en tan solo una década. Los hogares unipersonales habitados por solteros y casados tienden a mantenerse en cuanto a cifras. Por último, el subgrupo de los viudos descendió notoriamente (de $39,2 \%$ a 32,7 ) en el periodo de referencia. Este resultado señala WPS RI-SHUR, nำ, 2016, vol.1, ISSN: 2387-1768 


\section{WPSReview International on Sustainable \\ Housing and Urban Renewal (RI-SHUR)}

también que el aumento de la tasa de divorcio ha influido en el crecimiento de los hogares unipersonales en España en los últimos años. El boom del divorcio en España (2,3\% anual en 2015 según el Consejo General del Poder Judicial) tiene ya un efecto real y contundente sobre la morfología de nuestros hogares.

Tabla 5. El crecimiento de los hogares unipersonales y el cambio de la proporción en el estado civil en España (unidad: mil hogares, \%)

\begin{tabular}{l|l|l|l|l|l}
\hline \multicolumn{2}{l|}{} & \multicolumn{4}{l}{ Estado civil } \\
\cline { 3 - 6 } \multicolumn{2}{l|}{} & Soltero & Casado & Viudo & Divorciado \\
\hline 2001 & 42,1 & 14,8 & 39,4 & 3,7 \\
\hline 2011 & 44,3 & 13,4 & 32,5 & 9,8 \\
\hline $2001-$ & Crecimiento & 648 & 138 & 227 & 304 \\
\cline { 2 - 6 } 2011 & $\begin{array}{l}\text { Tasa de } \\
\text { crecimiento }\end{array}$ & 53,5 & 32,5 & 20,1 & 283,5 \\
\hline
\end{tabular}

Fuente: 2011 Censos de Población y Viviendas (España)

Gráfico 2. Pirámide de población de los hogares unipersonales con el estado civil por s exo y edad, 2011, España

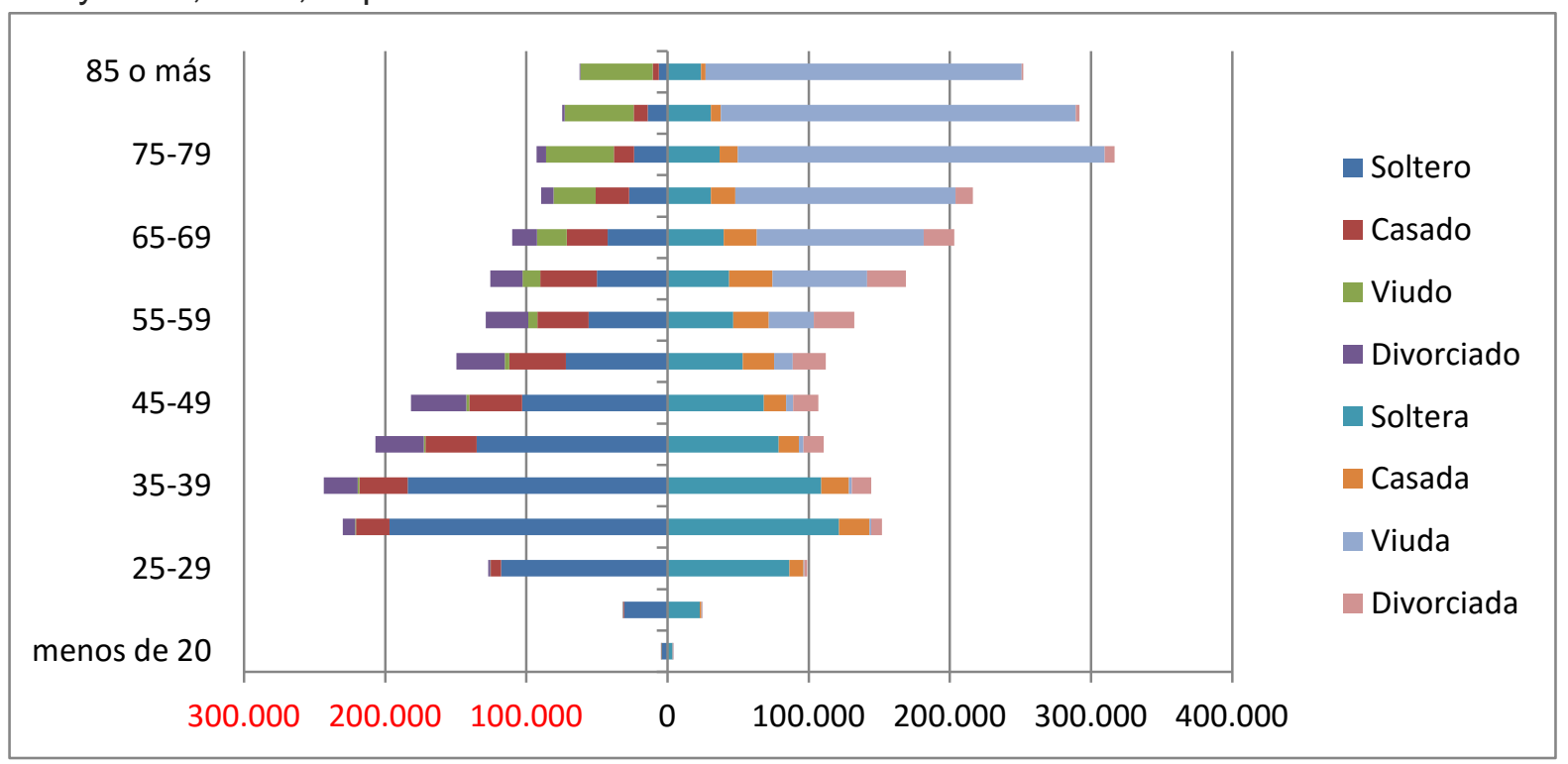

Fuente: Jeongju (2015) con datos del INE. 


\section{WPSReview International on Sustainable \\ Housing and Urban Renewal (RI-SHUR)}

Una revisión de la pirámide de los hogares unipersonales (gráfico 2) muestra un marcado sesgo hacia las mujeres viudas a partir de los 70 años. Esto refleja un rasgo demográfico ineludible (la destacada esperanza de vida en España -particularmente de las mujeres- ha potenciado su viudedad). Se da un segundo sesgo en la pirámide esta vez en el lado de los hombres- entre los solteros de 30 a 39 años. El dato refleja (1) la tardía emancipación del hogar familiar de los jóvenes y (2) la elevada edad de acceso al matrimonio (mayor en los hombres que en las mujeres: 34,5 frente a 32 años según el INE (2015.)

Un último rasgo a destacar de los hogares unipersonales en España de los últimos años es la imposición gradual de los sujetos de calificación universitaria frente al resto de grupos (Tabla 6). Estos hogares unipersonales aumentaron en 673000 en el periodo 2001-2011, con una tasa de crecimiento del $132,5 \%$ y representando, en la actualidad, el $28,2 \%$ sobre el total. Tal evolución no rompe la línea marcada por el resto de los países de la OCDE. Este nuevo perfil refleja facetas de la reciente evolución de nuestra sociedad como el aumento de sujetos que acceden a esta formación en su conjunto, así como su relación con el nivel de renta y los estilos de vida.

Tabla 6. El crecimiento de los hogares unipersonales por los niveles de educación, 200 1-2011, España

(unidad: mil hogares)

\begin{tabular}{l|l|l|l|l|l|l}
\hline & \multicolumn{3}{l}{2001} & 2011 & $2001-2011$ & \\
\cline { 2 - 7 } & Hogares & porcentaje & hogares & porcentaje & crecimiento & $\begin{array}{l}\text { Tasa de } \\
\text { crecimiento }\end{array}$ \\
\hline Total & 2,877 & 100 & 4,193 & 100 & 1,316 & 45.8 \\
\hline No estudio & 776 & 27.0 & 784 & 18.7 & 8 & 1.0 \\
\hline Primaria & 742 & 25.8 & 708 & 16.9 & -34 & -4.6 \\
\hline Secundaria & 514 & 17.9 & 897 & 21.4 & 383 & 74.5 \\
\hline Bachillerato & 338 & 11.7 & 624 & 14.9 & 286 & 84.6 \\
\hline $\begin{array}{l}\text { Universidad } \\
\text { O más }\end{array}$ & 508 & 17.7 & 1,181 & 28.2 & 673 & 132.5 \\
\hline
\end{tabular}

Fuente: Elaboración propia a partir de los Censos del INE (2011). 


\section{WPSReview International on Sustainable Housing and Urban Renewal (RI-SHUR)}

El tipo de relación inmobiliaria mantenida por los ocupantes de los hogares unipersonales es un dato de particular importancia para juzgar la estabilidad y prever la permanencia en el hogar de los sujetos. Considerando ahora la variable vivienda en propiedad, en España 3.186.000 hogares unipersonales están en propiedad (76 \%), por $616000(14,7 \%)$ en alquiler, mientras que el restante $9,3 \%$ está entre cesiones y otras formas (Tabla 7). Los datos son similares al conjunto de los hogares españoles. El predominio de la propiedad sobre el alquiler se presenta como un rasgo muy notable, no solo atribuible a los "solitarios", más bien se plasma en el conjunto del sistema. En este sentido el caso español es muy particular, de forma que las razones de la prevalencia del modelo de la propiedad se basa más en cuestiones culturales que económicas: el acceso a la vivienda propia como rito de paso y acceso a un nuevo estatus (Gil Calvo, 2002). Este modelo ha permanecido muy sólido en nuestra sociedad hasta la llegada de la crisis de 2008 , en la que las dificultades de pago hipotecario han ido en aumento (si bien, no son el cometido de este artículo) y las repercusiones sociales han alcanzado con fuerza el escenario político. Desde entonces la sociedad española apuesta por un giro hacia el parque de alquiler y la diversificación hacia otras fórmulas (difusión del alquiler con derecho a compra, por ejemplo). De hecho, ya se pueden constatar los primeros efectos estadísticos. En el caso de los hogares unipersonales se puede apreciar una caída notoria de la vivienda en propiedad en las nuevas generaciones: la vivienda propia en 2011 (tabla 8) estaba en un 59,5\% en el grupo de edad de $25-34$ años, significativamente por debajo del grupo de edad posterior: $72,7 \%$ (35-44 años).

Tabla 7. Tipo de vivienda ocupada por los hogares unipersonales, 2011 (unidad: mil hogares, \%)

\begin{tabular}{c|l|l|l|l|l}
\hline tipa & Total & Propia & Alquilada & Cedida gratis & Otra forma \\
\hline \multirow{2}{*}{ España } & 4.193 & 3.186 & 616 & 148 & 244 \\
& $(100)$ & $(76,0)$ & $(14,7)$ & $(3,5)$ & $(5,8)$ \\
\hline
\end{tabular}

Fuente: Elaboración propia a partir de los Censos de 2011.INE.

Tabla 8. Tipo de vivienda ocupada según edad por los hogares unipersonales de España, 2011

(unidad: mil hogares, \%)

WPS RI-SHUR, nํㅜ, 2016, vol.1, ISSN: 2387-1768 
WPSReview International on Sustainable

Housing and Urban Renewal (RI-SHUR)

\begin{tabular}{|l|l|l|l|l|}
\hline \multicolumn{1}{|c|}{ Tipo } & Propia & Alquilada & Cedida gratis & Otra forma \\
\hline \multirow{2}{*}{ 25-34 años } & 362 & 166 & 35 & 45 \\
& $(59,5)$ & $(27,4)$ & $(5,7)$ & $(7,4)$ \\
\hline \multirow{2}{*}{ 35-44 años } & 513 & 122 & 27 & 43 \\
& $(72,7)$ & $(17,3)$ & $(3,9)$ & $(6,1)$ \\
\hline \multirow{2}{*}{$45-54$ años } & 399 & 92 & 22 & 38 \\
& $(72,5)$ & $(16,7)$ & $(3,9)$ & $(6,8)$ \\
\hline \multirow{2}{*}{$55-64$ años } & 445 & 68 & 14 & 29 \\
& $(80,0)$ & $(12,3)$ & $(2,5)$ & $(5,2)$ \\
\hline \multirow{2}{*}{$65-74$ años } & 523 & 55 & 13 & 27 \\
& $(84,6)$ & $(8,9)$ & $(2,1)$ & $(4,4)$ \\
\hline \multirow{2}{*}{ 75-84 años } & 659 & 62 & 19 & 37 \\
& $(84,9)$ & $(8,0)$ & $(2,4)$ & $(4,7)$ \\
\hline \multirow{2}{*}{ 85 o más años } & 258 & 26 & 12 & 19 \\
& $(82,0)$ & $(8,4)$ & $(3,7)$ & $(5,9)$ \\
\hline
\end{tabular}

Fuente: Elaboración propia a partir de los Censos de 2011. INE.

¿En qué medida crecerán los hogares unipersonales en España?, las proyecciones de los hogares responde a esta pregunta. Según las proyecciones realizadas por el INE hasta el año 2029, el número de los hogares totales alcanzará la cifra de 19.204.058 y el de los hogares unipersonales alcanzará 5.711.125 correspondiente al $29,7 \%$ en España. (Tabla 9). Es decir el aumento es significativo tanto en términos proporcionales (de 25\% a 29,7\%) como considerando la cifra total ( 1.300 .000 hogares más). Asimismo, podemos apreciar como el tamaño medio del hogar no frenará en su descenso. Según el INE el hogar tipo descenderá de 2,52 miembros en 2014 a 2,34 en 2029 (ver tablas 9 y 10). Observamos, por lo tanto, que la transformación implicada en nuestra sociedad es algo muy tangible tanto en términos sociológicos como en la propia estructura urbana: estaremos más dispersos y menos acompañados. Esta evolución transformará las políticas sociales del futuro (soledad, dificultades en los cuidados de la tercera edad o problemas económicos para afrontar la renta: vivir más "fragmentados" sale más caro). 


\section{WPSReview International on Sustainable \\ Housing and Urban Renewal (RI-SHUR)}

Tabla 9. Proyección de Hogares (1 persona) 2014-2029

\begin{tabular}{l|l|l|l|l}
\hline \multirow{2}{*}{} & \multicolumn{2}{|l|}{2014} & \multicolumn{2}{l}{2029} \\
\cline { 2 - 5 } & Total hogares & 1 persona (\%) & Total hogares & 1 persona (\%) \\
\hline España & 18.252 .887 & $4.480 .391(24.5)$ & 19.204 .058 & $5.711 .125(29.7)$ \\
\hline
\end{tabular}

Fuente: Proyección de Hogares en España (INE)

Tabla 10. Evolución proyectada del tamaño medio del hogar

\begin{tabular}{l|l|l|l|l|l|l}
\hline & 2014 & 2017 & 2020 & 2023 & 2026 & 2029 \\
\hline España & 2,52 & 2,48 & 2,45 & 2,41 & 2,37 & 2,34 \\
\hline
\end{tabular}

Fuente: Proyección de Hogares en España (INE)

Una reciente investigación (Jeoongju, 2015) comparaba la evolución de España y Core a en los hogares unipersonales. El investigador argumentaba el sentido de tal compara ción resaltando los interesantes paralelismos entre ambos países tanto demográficos ( similar población, proporción de hogares unipersonales o tamaño medio del hogar) co mo en términos sociales (acceso tardío a la modernidad). "La estructura de los hogare s está cambiando hacia hogares pequeños. Los hogares unipersonales siguen aument ando en ambos países, si bien el ritmo es mayor en Corea que ya supera a España y $\mathrm{s}$ e distanciará en el futuro. Esto afecta a aspectos sociológicos tan importantes como lo s servicios de bienestar, el consumo, modelos residenciales y adaptación a la economí a a los solitarios. Abordando el factor edad, los jóvenes están retrasando el matrimonio por la crisis económica (causa dominante en Corea) y los divorcios y separaciones en la mediana edad está creciendo rápidamente (causa dominante en España) ante una v isión cambiante de la pareja y la familia, con tendencias marcadamente individualistas en ambos países. Además, ambas sociedades sufren importantes problemas por la pr oliferación de hogares unipersonales en la tercera edad (fenómeno relacionado con el aumento de la esperanza de vida). Las diferencias son mínimas: tan solo destacar una mayor presencia de los mayores de 75 en España. Esto plantea importantes dificultad es sociales en ambos países, si bien estas son mayores en Corea por las insuficientes coberturas de la Seguridad Social, deficiencias del Estado de Bienestar y las dificultad es para acceder a la vivienda en propiedad (tasa muy alta -por el contrario- en el caso español)" (Jeonju, 2015: 23-24). Asimismo, el autor observa en ambas sociedades not

WPS RI-SHUR, nำ, 2016, vol.1, ISSN: 2387-1768 


\section{WPSReview International on Sustainable \\ Housing and Urban Renewal (RI-SHUR)}

orias cifras de "solitarios" en las edades próximas a la treintena en ambos casos. Sin e mbargo las causas no son las mismas: "en el caso coreano se ha dado una fuerte influ encia de los casos en los que las bodas se aplazan hasta consolidar la situación econó mica de los cónyuges, afectando en menor medida el divorcio, mientras que en Españ a, separaciones, divorcios o solteros de larga duración han hecho crecer este conglom erado" (Ibíd., 2015: 24). Por último el autor destaca como rasgo muy notorio de nuestr a estructura de hogares la permanencia del modelo de propiedad sobre el alquiler, dad as las diferencias de tradición y cultura entre ambas sociedades (el caso coreano se $\mathrm{m}$ uestra más cercano a los estándares de la OCDE).

\section{La institución familiar, las redes interpersonales y los hogares unipersonales en España}

En la formación de nuestros hogares unipersonales se manifiesta como rasgo esencial una edad tardía en su constitución (observemos en la pirámide anteriorgráfico 2- como el "ensanche" se produce pasada la treintena), algo más tarde de lo marcado por los patrones para los países occidentales. En este sentido, la precariedad laboral ha condicionado la salida de los jóvenes del hogar familiar: "Este síndrome de retraso alcanza sus tasas más altas en España e Italia, cuyos jóvenes permanecen en casa más tiempo con respecto al pasado y en relación a sus coetáneos europeos" (Arias-Aparicio, 2013: 156). No podemos cuestionar que nuestra marcada tradición familista ha tenido una gran influencia en la configuración del escenario actual. La familia española salió al paso para compensar el desplome del mercado de trabajo en calidad y cantidad (desempleo) (Gil Calvo, 2002). La permanencia de la generación $Y$ (nacidos desde 1985) en el hogar familiar -mayor que la de cualquier generación anterior- marcará a sus jóvenes para siempre. Nuestra más sólida institución cubre los aspectos que el ámbito público es incapaz de solventar: servicios de cuidado de enfermos y/o mayores, y transferencias para apoyar la transición de los jóvenes (Flaquer, 2004). Los propios jóvenes son cada vez más participativos en el apoyo familiar, actuando tanto como receptores como prestadores (Ayuso, 2012).Aunque esta diversidad de funciones sea un rasgo positivo de nuestra sociedad, puede relajar la presión sobre otras instituciones del Estado de Bienestar, perjudicando particularmente a los jóvenes de inferior clase social (Gentile, 2010). .

WPS RI-SHUR, n3, 2016, vol.1, ISSN: 2387-1768 


\section{WPSReview International on Sustainable \\ Housing and Urban Renewal (RI-SHUR)}

Por lo tanto, el hecho de no alcanzar aún la tasa de hogares unipersonales de la OCDE $(27 \%$, por $25 \%)$ no viene explicada por una cuestión de evolución de los valores y/o de acceso a la modernidad, más bien, la explicación se haya en cuestiones muy pragmáticas: los jóvenes tardan en emanciparse por las hostilidades que presenta nuestro mercado laboral y, al mismo tiempo, encuentran en la familia un refugio que les permite aplazar la formación del primer hogar (unipersonal en la mayoría de los casos). Como resaltara Flaquer (2004) la familia, en su papel habitual de institución fuerte en España, contribuye a paliar los efectos de la precariedad laboral y asume problemas que, ni el sector público ni el privado, pueden resolver en nuestros tiempos (aunque paguen sus impuestos para ello).

Asimismo, para los investigadores sociales resulta sencillo respaldar la hipótesis de que los "solitarios" mostrarán menos apego a las redes familiares y de amistad, dada la tentación de asociar el solitario en el hogar con el sujeto de naturaleza solitaria. Sin embargo, un reciente estudio publicado por Barroso et al. (2016) nos induce a tratar el tema con enorme prudencia. Tras aplicar diversos modelos de regresión logística no encontraron asociación significativa entre la vida en solitario y un menor aprecio de las relaciones con la familia y los amigos. Tan solo se encontró un menor apego en el grupo de edad de 55 a 64 años (utilizando datos de OPAFAM, 2014). Sin embargo los investigadores explican el fenómeno en base a un factor coyuntural: en estas edades el solitario asume la situación de "nido vacío" (desaparecen las expectativas de proyectos futuros de convivencia y/o se encuentran salientes de experiencias de cohabitación frustradas: relaciones sentimentales sin proyección, divorcios o separaciones). En definitiva, el solitario español muestra el mismo apego hacia la familia y los amigos que el resto de la población, valorando igualmente sus redes interpersonales y el poder rodearse de la gente que aprecia.

\section{Conclusiones y propuestas}

En España se ha dado un continuo incremento de los hogares unipersonales en los últimos 40 años. Esta tendencia se ha mantenido incluso desde la llegada de la crisis económica de 2008. Estos constituían en 2014 una cuarta parte del total de los hogares registrados. En este sentido nuestro país cumple los patrones de la sociedad occidental, con un comportamiento que está en la línea de los patrones de la WPS RI-SHUR, no3, 2016, vol.1, ISSN: 2387-1768 


\section{WPSReview International on Sustainable \\ Housing and Urban Renewal (RI-SHUR)}

modernidad (individuación y procesos de fragmentación familiar, particularmente). Sin embargo España está aún por debajo del promedio de la OCDE, y tal resistencia a rebasarlo está vinculada a un modelo familista que permite a los jóvenes una emancipación tardía, que a su vez están condicionados por una compleja inserción laboral. Aun así la progresión de estos hogares continuará firme, acercándose a los 6 millones en 2029 (según las proyecciones del Ine) frente a los 4,2 de 2014.

Acerca de los habitantes de estos hogares solitarios, las mujeres mayores de 65 constituyen el subgrupo más numeroso (viudedad y soledad de los ancianos). Debemos resaltar otro subgrupo que destaca por su peso en la pirámide -los varones entre 30 y 39 años-, se trata de un colectivo que recoge, sobre todo, solteros que aún no han accedido al matrimonio o a uniones de hecho y que en muchos casos padecen dificultades económicas y laborales para afrontar un nuevo proyecto familiar (este patrón coincide con el grupo de edad anterior en la mayoría de países de la OCDE). Separados y divorciados engrosan también este grupo, aunque en menor proporción.

Los datos de hogares en su conjunto reflejan un proceso de atomización del modelo residencial español. En 2014 entre los hogares de una y dos personas se cubría casi un $60 \%$ sobre el total. Desde una perspectiva, tanto sociológica como económica, este se convierte en un rasgo esencial de nuestro modelo de sociedad, hoy en día, en proceso de rediseño de gran parte de sus servicios. Debemos considerar que tan solo cuatro décadas atrás nuestros hogares estaban habitados por extensas unidades familiares que implicaban diferentes demandas y, por lo tanto, distintos servicios.

La inmensa mayoría de los hogares unipersonales están en régimen de propiedad, con cifras muy similares al conjunto de los hogares (cerca del 85\% en propiedad). Sin duda en este aspecto incide nuestra tradición y una particular cultura de la propiedad. Sin embargo la caída de la propiedad y la consecuente emergencia de los alquileres se manifiestan en los grupos de edad inferiores. Por debajo de los 40 años, el nuevo comportamiento se muestra como síntoma de una posible transición, si bien el dato viene muy condicionado por la repercusión de una crisis económica que tiene una particular "lectura inmobiliaria". El hogar unipersonal del futuro mostrará un mayor equilibrio entre los regímenes de propiedad y alquiler, además de acogerse a las

WPS RI-SHUR, no3, 2016, vol.1, ISSN: 2387-1768 


\section{WPSReview International on Sustainable \\ Housing and Urban Renewal (RI-SHUR)}

nuevas alternativas o fórmulas mixtas.

Abordando las repercusiones psicosociales del fenómeno, las aproximaciones recientes (Barroso et al. 2016) nos llevan a ser muy prudentes con la formación del estereotipo del "lobo solitario". Las personas que viven solas muestran tanto apego a sus familiares y amigos como los demás y le dan idéntica importancia a la solidez de sus relaciones sociales. El solitario en España, por lo tanto, está más condicionado por un conjunto de condiciones socioeconómicas y tendencias que le "arrastran" hacia el modelo (muy variables según género y edad), que por una actitud desocializadora. La soledad en el hogar no implica ni refleja algo innato en el sujeto. Debemos considerar fenómenos como la individuación o las relaciones líquidas -ineludibles consecuencias de la modernidad- como fuentes tendencias sociológicas de alcance colectivo que nos envuelven, más allá de actitudes particulares.

Como propuestas a considerar para posteriores intervenciones, debo resaltar (1) la tardía edad a la que los jóvenes acceden al hogar unipersonal y su lento proceso de salida constituyen la enésima llamada de atención a la precariedad de nuestro mercado de trabajo; (2) la particular situación de los ancianos como ocupantes predominantes de los hogares solitarios -mujeres, sobre todo- tiene una directa afección tanto las políticas sociales del futuro como a la oferta de servicios privados; y (3) la atomización de los hogares españoles nos lleva a replantear el concepto de vivienda, así como la gestión de los espacios urbanos, que deben orientarse cada vez más a facilitar que nos sintamos (o estemos) acompañados.

\section{Referencias bibliográficas}

Arias-Aparicio, F. (2013). "Inestabilidad laboral y emancipación. Jóvenes-adultos en el umbral del mileurismo en Barcelona y Roma" (Crítica al libro de A. Gentile). Revista Española de Investigaciones Sociológicas, no 144, pp.153-164.

Ayuso Sánchez, L. (2012). "El deber de apoyar a la familia. Una revisión del pacto intergeneracional de ayudas familiares en España". Panorama Social, oㅡ 15, pp. 143158.

Barroso, I., Cámara, A., Rodríguez, C. y Morente, F. (2016). ¿Refugio de afectos? La familia vista por los ocupantes de los hogares solitarios. XII Congreso Español de Sociología: Grupo 3; comunicación.

WPS RI-SHUR, ํㅜ, 2016, vol.1, ISSN: 2387-1768 


\section{WPSReview International on Sustainable \\ Housing and Urban Renewal (RI-SHUR)}

Bauman, Z. (2005). Amor líquido. Acerca de la fragilidad de los vínculos humanos. Madrid: Fondo de Cultura Económica.

Beck, U. (2002). La sociedad del riesgo. Barcelona. Paidós.

Becker, G. (1987). Tratado sobre la familia, Madrid, Alianza.

Flaquer Vilardebó, LI. (2004). "La articulación entre familia y Estado de Bienestar en los países de la Europa del sur", Papers, 73 (1), pp. 27-58.

Gentile, A. (2010). "De vuelta al nido en tiempo de crisis. Los boomerang kids españoles". Revista de Estudios de Juventud, 90, 181-203.

Gil Calvo, E. (2002). "Emancipación tardía y estrategia familiar". Revista de Estudios de Juventud. 58: 1-9.

Jeongju, L. (2015). El crecimiento de los hogares personales en Corea y España. Trabajo Fin de Máster; Máster de Sociología Aplicada. Universidad de Málaga.

Roussel, L. (1986). "Evolution récente de la structure des ménages dans quelques pays industriéis", Population, 6: 913-93.

Sennet, R. (2000). La corrosión del carácter. Madrid: Alianza 